\title{
QUEEN'S
UNIVERSITY
BELFAST
}

\section{Reversible Photocontrol of Deoxyribozyme-Catalyzed RNA Cleavage under Multiple-Turnover Conditions}

Keiper, S., \& Vyle, J. (2006). Reversible Photocontrol of Deoxyribozyme-Catalyzed RNA Cleavage under Multiple-Turnover Conditions. Angewandte Chemie International Edition, 45(20), 3306-3309.

https://doi.org/10.1002/anie.200600164

\section{Published in:}

Angewandte Chemie International Edition

\section{Document Version:}

Peer reviewed version

Queen's University Belfast - Research Portal:

Link to publication record in Queen's University Belfast Research Portal

\section{General rights}

Copyright for the publications made accessible via the Queen's University Belfast Research Portal is retained by the author(s) and / or other copyright owners and it is a condition of accessing these publications that users recognise and abide by the legal requirements associated with these rights.

Take down policy

The Research Portal is Queen's institutional repository that provides access to Queen's research output. Every effort has been made to ensure that content in the Research Portal does not infringe any person's rights, or applicable UK laws. If you discover content in the Research Portal that you believe breaches copyright or violates any law, please contact openaccess@qub.ac.uk. 


\section{Angewandte}

Supporting Information

(c) Wiley-VCH 2006

69451 Weinheim, Germany 


\title{
Reversible photocontrol of deoxyribozyme-catalyzed RNA cleavage under multiple turnover conditions
}

\author{
Sonja Keiper and Joseph S. Vyle*
}

\author{
Department of Chemistry and Chemical Engineering, David Keir Building, Stranmillis Road, \\ Belfast BT9 5AG, UK
}

\section{Synthesis of phosphoramidites}

Dichloromethane (DCM) was dried under reflux over calcium hydride, distilled and stored over activated $3 \AA$ molecular sieves under argon. Triethylamine and $n$-butylamine were dried under reflux over calcium hydride and distilled immediately prior to use; $N, N$-diisopropylethylamine (DIPEA) was similarly purified but stored over activated $3 \AA$ molecular sieves for upto 6 weeks. DMF was obtained as anhydrous solvent over molecular sieves from Fluka. Dry $\mathrm{MeOH}$ was obtained by distillation from magnesium turnings and stored over activated $3 \AA$ molecular sieves. Tetrahydrofuran (THF) was distilled from sodium/benzophenone, stored over activated $3 \AA$ molecular sieves and used within 7 days. Acid-free ethyl acetate was prepared by prewashing with saturated $\mathrm{Na}_{2} \mathrm{CO}_{3}$. $\mathrm{CDCl}_{3}$ was passed through activated basic $\mathrm{Al}_{2} \mathrm{O}_{3}$ immediately prior to use and NMR's obtained within 1 hour $\left({ }^{31} \mathrm{P},{ }^{13} \mathrm{C}\right)$ or 12 hours $\left({ }^{1} \mathrm{H}\right)$ of the $\mathrm{CDCl}_{3}$ solution being prepared. All other reagents were purchased from commercial suppliers and used without further purification. Silica gel (particle size 30-60 $\mu \mathrm{m}$ ) for flash column chromatography was obtained from $\mathrm{BDH}$ and dried at $300^{\circ} \mathrm{C}$ immediately prior to use.

UV-Visible data were obtained with a VARIAN CARY 100 Spectrometer. Nuclear Magnetic Resonance (NMR) spectra were run on Bruker AC-250 and AMX-400 spectrometers.

$o$-Phenylazobenzoic acid- $N$-hydroxysuccinimidyl ester.

To a stirred suspension of $o$-phenylazobenzoic acid $(452 \mathrm{mg}, 2.0 \mathrm{mmol})$ and $N$ hydroxysuccinimide (255 mg, $2.2 \mathrm{mmol})$ in anhydrous DCM (10 ml) $N, N^{\prime}$ dicyclohexylcarbodiimide (454 mg, $2.2 \mathrm{mmol}$ ) was added at room temperature under argon. After $45 \mathrm{~min}$, precipitated $N, N^{\prime}$-dicyclohexylurea was removed by filtration and solvent removed in vacuo. The residual brown solid was purified by silica gel column chromatography eluting with anhydrous DCM to yield the<smiles>O=C(ON1C(=O)CCC1=O)c1ccccc1N=Nc1ccccc1</smiles>
product as a moisture-sensitive brown-orange solid $(565 \mathrm{mg}, 87 \%) .{ }^{1} \mathrm{H} \mathrm{NMR}\left(300 \mathrm{MHz}, \mathrm{CDCl}_{3}, 20^{\circ} \mathrm{C}, \mathrm{TMS}\right) \delta=$ $2.92\left(4 \mathrm{H}, \mathrm{s}, 2\right.$ x $\left.\underline{\mathrm{CH}}_{2}\right), 7.50$ - $7.60\left(4 \mathrm{H}, \mathrm{m}, \underline{\mathrm{H}} 3, \underline{\mathrm{H}} 4, \underline{\mathrm{H}} 5, \mathrm{PhN}_{2} ; \underline{\mathrm{H}} 4 \mathrm{ArCO}\right), 7.74$ (2H, m, $\left.\underline{\mathrm{H}} 2, \underline{\mathrm{H}} 6 \mathrm{PhN}_{2}\right), 8.06$ (3H, $\mathrm{m}, \underline{\mathrm{H}} 3, \underline{\mathrm{H}} 5, \underline{\mathrm{H}} 6 \mathrm{ArCO}) ;{ }^{13} \mathrm{C}\left(76 \mathrm{MHz}, \mathrm{CDCl}_{3}, 20^{\circ} \mathrm{C}\right.$, TMS) $\delta=26.2,119.0,124.2,124.40,129.6,130.5,131.3$, $132.3,134.3,152.7,152.9,153.0,169.4 ; \mathrm{ES}+\mathrm{MS} \mathrm{m} / \mathrm{z}=324(\mathrm{M}+\mathrm{H}), 346(\mathrm{M}+\mathrm{Na})$

5'-O-(4,4'-dimethoxytrityl)-2'-( $N$-(o-phenylazo)benzamido)-2'-deoxyuridine.

To a stirred solution of $o$-phenylazobenzoic acid- $N$-hydroxysuccinimidyl ester (260 $\mathrm{mg}, 0.80 \mathrm{mmol})$ in anhydrous DMF $(5 \mathrm{ml})$ at room temperature was added $n$-butylamine $(10 \mu \mathrm{l}, 0.10 \mathrm{mmol})$ and the reaction mixture maintained under these conditions for a further $10 \mathrm{~min}$. This solution was transferred under argon to a flask containing 5 '- $O$-(4,4'-dimethoxytrityl)-2'-amino-2'-deoxyuridine (250 $\mathrm{mg}, 0.46 \mathrm{mmol}$ ) and $N, N$-diisopropylethylamine (DIPEA: $80 \mu \mathrm{l}, 0.47 \mathrm{mmol}$ ) added. The reaction was stirred under ambient conditions for a further 6 hours, a further aliquot of DIPEA (100 $\mu 1,0.59 \mathrm{mmol})$ was added and the reaction stored

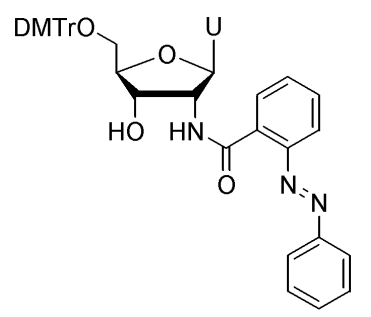
at $3{ }^{\circ} \mathrm{C}$ overnight. Excess activated ester was quenched by addition of $n$-butylamine $(190 \mu 1,1.92 \mathrm{mmol})$ and stirring at room temperature for 1 hour. The reaction mixture was diluted with ethyl acetate $(250 \mathrm{ml})$ and washed successively with aqueous saturated $\mathrm{NaHCO}_{3}(2 \times 150 \mathrm{ml})$ and saturated $\mathrm{NaCl}(2 \times 150 \mathrm{ml})$. The organics were dried over anhydrous sodium sulfate, filtered and solvent removed in vacuo. The resultant red gum was purified by silica gel column chromatography, eluting with a gradient of $0-2 \%(\mathrm{v} / \mathrm{v})$ methanol in DCM containing $1 \%$ (v/v) triethylamine to give the title compound as an orange solid $(250 \mathrm{mg}, 72 \%)$. ${ }^{1} \mathrm{H} \mathrm{NMR} \mathrm{(300} \mathrm{MHz,} \mathrm{CDCl}_{3}$, $\left.20^{\circ} \mathrm{C}, \mathrm{TMS}\right) \delta=3.42\left(2 \mathrm{H}, \psi \mathrm{d}, \underline{\mathrm{H}} 5^{\prime}, \underline{\mathrm{H}} 5^{\prime}\right.$ ') $3.79\left(6 \mathrm{H}, \mathrm{s}, 2 \mathrm{x} \mathrm{C} \underline{\mathrm{H}}_{3} \mathrm{O}\right), 4.17\left(1 \mathrm{H}, \mathrm{m}, \underline{\mathrm{H}} 4^{\prime}\right), 4.52\left(1 \mathrm{H}, \mathrm{dd},{ }^{3} J(\mathrm{H}, \mathrm{H})=\right.$ $\left.1.8,5.7 \mathrm{~Hz}, \underline{\mathrm{H}} 3^{\prime}\right), 5.03\left(1 \mathrm{H}, \mathrm{m}, \underline{\mathrm{H}} 2^{\prime}\right), 5.45\left(1 \mathrm{H}, \mathrm{d}^{3} J(\mathrm{H}, \mathrm{H})=8.1 \mathrm{~Hz}, \underline{\mathrm{H}} 5\right), 6.18\left(1 \mathrm{H}, \mathrm{d}^{3} J(\mathrm{H}, \mathrm{H})=7.8 \mathrm{~Hz}, \underline{\mathrm{H}} 1^{\prime}\right)$, $6.85\left(4 \mathrm{H}, \mathrm{d}^{3} J(\mathrm{H}, \mathrm{H})=8.4 \mathrm{~Hz}, \mathrm{DMTr} \underline{\mathrm{H}} o\right.$ to $\left.\mathrm{OMe}\right), 7.29-7.60(14 \mathrm{H}, \mathrm{m}, \mathrm{DMTr}-\mathrm{H}, \mathrm{AB}-\underline{\mathrm{H}}), 7.70\left(1 \mathrm{H}, \mathrm{d}^{3} J(\mathrm{H}, \mathrm{H})\right.$ $=8.1 \mathrm{~Hz}, \underline{\mathrm{H}} 6), 7.77(1 \mathrm{H}, \mathrm{m}, \mathrm{AB}-\underline{\mathrm{H}}), 7.96(2 \mathrm{H}, \mathrm{m}, \mathrm{AB}-\underline{\mathrm{H}}), 8.31(1 \mathrm{H}, \mathrm{m}, \mathrm{AB}-\underline{\mathrm{H}}), 9.17\left(1 \mathrm{H}, \mathrm{d}{ }^{3} J(\mathrm{H}, \mathrm{H})=7.8 \mathrm{~Hz}\right.$, $\mathrm{AB}-\underline{\mathrm{H}}) ;{ }^{13} \mathrm{C} \mathrm{NMR}\left(126 \mathrm{MHz}, \mathrm{CDCl}_{3}, 20^{\circ} \mathrm{C}, \mathrm{TMS}\right) \delta=54.3,55.6,62.8,70.2,84.5,86.0,86.2,101.7,112.3$, 115.2, 122.1, 122.7, 126.1, 127.0, 127.2, 128.4, 128.4, 129.2, 129.6, 130.3, 130.4, 131.0, 131.2, 134.3, 134.5, 139.5, 143.3, 148.8, 149.8, 151.3, 157.7, 162.2, 165.6; HRMS (ESI) 753.2789 (calc.753.2799 for $\mathrm{MH}^{+}$). 
5'-O-(4,4'-dimethoxytrityl)-2'-( $N$-(o-phenylazo)benzamido)-2'-deoxyuridine-3'- $O$-(2-cyanoethyl- $N, N$ diisopropyl-amino)-phosphoramidite.

To a stirred solution of 5'-O-(4,4'-dimethoxytrityl)-2'-( $N$ - $(\mathrm{O}$ phenylazo)benzamido)-2'-deoxyuridine (180 $\mathrm{mg}, 0.24 \mathrm{mmol})$ and DIPEA $(90 \mu \mathrm{l}$, $0.98 \mathrm{mmol})$ in anhydrous DCM $(4 \mathrm{ml})$ and THF $(1 \mathrm{ml})$ at room temperature was added 2-cyanoethyl- $N, N$-diisopropylaminochlorophosphoramidite $(86 \mu 1,0.53$ $\mathrm{mmol}$ ) dropwise over $3 \mathrm{~min}$. The reaction was maintained under these conditions for a further $30 \mathrm{~min}$ and then quenched following addition of anhydrous methanol $(60 \mu \mathrm{l}, 1.48 \mathrm{mmol})$ and DIPEA $(60 \mu \mathrm{l}, 0.65 \mathrm{mmol})$ and stirring for a further $10 \mathrm{~min}$. The reaction mixture was diluted with acid-free ethyl acetate (50 $\mathrm{ml})$ and washed successively with aqueous saturated $\mathrm{NaHCO}_{3}(2 \times 50 \mathrm{ml})$,

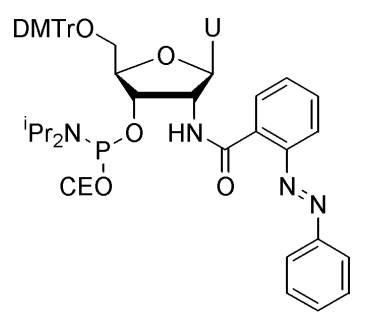
saturated $\mathrm{NaCl}(1 \times 50 \mathrm{ml})$. The organics were dried over anhydrous sodium sulfate, filtered and solvent removed in vacuo. The resultant solid was purified by silica gel column chromatography, eluting with $2 \%$ (v/v) solutions of triethylamine in 3:1 DCM:40-60 petroleum ether; pure DCM and 3:1 DCM : ethyl acetate. The title compound was isolated as a brown foam (194 mg, $0.20 \mathrm{mmol}, 85 \%)$. ${ }^{1} \mathrm{H} \mathrm{NMR}\left(300 \mathrm{MHz}, \mathrm{CDCl}_{3}, 20^{\circ} \mathrm{C}, \mathrm{TMS}\right) \delta=0.80-$

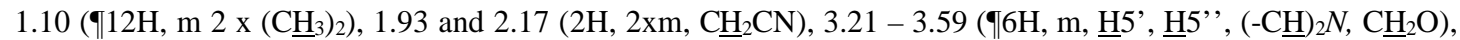
$3.81\left(6 \mathrm{H}, 2 \mathrm{xs}, 2 \mathrm{xCH}_{3} \mathrm{O}\right), 4.25$ and $4.37\left(1 \mathrm{H}, 2 \mathrm{xs}, \underline{\mathrm{H}} 4^{\prime}\right), 4.55$ and $4.73\left(1 \mathrm{H}, 2 \mathrm{xdd}, 5.0,11.4,5.3,10.6, \underline{\mathrm{H}} 2^{\prime}\right), 5.23$ and $5.32\left(1 \mathrm{H}, 2 x m, \underline{H} 3^{\prime}\right), 5.43\left(1 \mathrm{H}, \mathrm{d}^{3} J(\mathrm{H}, \mathrm{H})=8.2 \mathrm{~Hz}, \underline{\mathrm{H}} 5\right), 6.34\left(1 \mathrm{H}, \psi \mathrm{t},{ }^{3} J(\mathrm{H}, \mathrm{H})=8.2 \mathrm{~Hz}, \underline{\mathrm{H}}{ }^{\prime}{ }^{\prime}\right), 6.88(4 \mathrm{H}, \mathrm{d}$ ${ }^{3} J(\mathrm{H}, \mathrm{H})=8.9 \mathrm{~Hz}, \mathrm{DMTr} \underline{\mathrm{H}} o-$ to OMe), $7.30-7.70(\mathbb{I} 12 \mathrm{H}, \mathrm{m}, \mathrm{DMTr} \underline{\mathrm{H}}, \mathrm{NHC}(\mathrm{O}) \mathrm{AB} \mathrm{AB}-\underline{\mathrm{H}}) 7.73$ and $7.79(1 \mathrm{H}$, $\left.2 \mathrm{xd}^{3} J(\mathrm{H}, \mathrm{H})=9.3,9.3 \mathrm{~Hz}, \underline{\mathrm{H}} 6\right), 7.87(1 \mathrm{H}, \mathrm{m}, \mathrm{AB}-\underline{\mathrm{H}}) 8.00(2 \mathrm{H}, \mathrm{m}, \mathrm{AB}-\underline{\mathrm{H}}) 8.36$ and $8.40(1 \mathrm{H}, 2 \mathrm{xm}, \mathrm{AB}-\underline{\mathrm{H}}), 8.85$ and $9.06\left(1 \mathrm{H}, 2 \mathrm{xd},{ }^{3} \mathrm{~J}(\mathrm{H}, \mathrm{H})=8.2 \mathrm{~Hz} \mathrm{AB}-\underline{\mathrm{H}}\right) ;{ }^{31} \mathrm{P}$ NMR $\left(121 \mathrm{MHz}, \mathrm{CDCl}_{3}, 20^{\circ} \mathrm{C}, 85 \% \mathrm{H}_{3} \mathrm{PO}_{4}\right) \delta=149.6,147.9$

m-Phenylazobenzoic acid- $N$-hydroxysuccinimidyl ester.

To a stirred suspension of $m$-phenylazobenzoic acid $(452 \mathrm{mg}, 2.0 \mathrm{mmol})$ and $N$-hydroxysuccinimide (255 mg, $2.2 \mathrm{mmol})$ in anhydrous DCM (10 ml) N,N'dicyclohexylcarbodiimide ( $454 \mathrm{mg}, 2.2 \mathrm{mmol}$ ) was added at room temperature under argon. After $45 \mathrm{~min}$, the precipitated urea was removed by filtration and<smiles>O=C(ON1C(=O)CCC1=O)c1cccc(N=Nc2ccccc2)c1</smiles>
the solvent removed in vacuo. The residual orange solid was purified by silica gel column chromatography eluting with anhydrous DCM to yield the pure product as a moisture-sensitive orange solid $(612 \mathrm{mg}, 95 \%)$. ${ }^{1} \mathrm{H}$ NMR $\left(300 \mathrm{MHz}, \mathrm{CDCl}_{3}, 20^{\circ} \mathrm{C}\right.$, TMS $) \delta=2.95\left(4 \mathrm{H}, \mathrm{s}, 2 \mathrm{x} \mathrm{C \underline {H } _ { 2 }}\right), 7.53(3 \mathrm{H}, \mathrm{m}, \mathrm{Ph} \underline{\mathrm{H}}), 7.67\left(1 \mathrm{H}, \mathrm{t}{ }^{3} \mathrm{~J}(\mathrm{H}, \mathrm{H})=7.8\right.$ $\mathrm{Hz}, \operatorname{Ar} \underline{\mathrm{H}} \mathrm{m}$ to $\left.\mathrm{N}_{2}\right), 7.97\left(2 \mathrm{H}, \mathrm{m}, \mathrm{Ph} \underline{\mathrm{H}} o\right.$ to $\left.\mathrm{N}_{2}\right), 8.24\left(2 \mathrm{H}, \mathrm{dd} J=1.5,6.0 \mathrm{~Hz}, \operatorname{Ar} \underline{\mathrm{H}} o, p\right.$ to $\left.\mathrm{C}(\mathrm{O}), \mathrm{N}_{2}\right), 8.69(1 \mathrm{H}, \mathrm{s}$, $\operatorname{ArH} o$ to $\left.\mathrm{C}(\mathrm{O}), \mathrm{N}_{2}\right) ;{ }^{13} \mathrm{C} \mathrm{NMR}\left(76 \mathrm{Mz}, \mathrm{CDCl}_{3}, 20^{\circ} \mathrm{C}\right.$, TMS $) \delta=26.1,123.5,123.6,125.3,130.0,130.2,130.4$, 132.6, 133.0, 152.7, 153.0, 161.4, 169.6; MS (ES+) m/z = $324(\mathrm{M}+\mathrm{H}), 346(\mathrm{M}+\mathrm{Na})$

5'-O-(4,4'-dimethoxytrityl)-2'-( $N$-(m-phenylazo)benzamido)-2'-deoxyuridine.

To a stirred solution of $m$-phenylazobenzoic acid- $N$ hydroxysuccinimidyl ester $(260 \mathrm{mg}, 0.80 \mathrm{mmol})$ in anhydrous DMF (5 $\mathrm{ml})$ at room temperature was added $n$-butylamine $(10 \mu \mathrm{l}, 0.10 \mathrm{mmol})$ and the reaction mixture maintained under these conditions for a further $10 \mathrm{~min}$. The reaction mixture was transferred under argon to a flask containing 5 '- $O$-(4,4'-dimethoxytrityl)-2'-amino-2'-deoxyuridine<smiles>COCC(O)C1OC(Cl)C1NC(=O)c1cccc(N=Nc2ccccc2)c1</smiles>
$(250 \mathrm{mg}, 0.46 \mathrm{mmol}$ ) and $N, N$-diisopropylethylamine (DIPEA: $80 \mu 1,0.47 \mathrm{mmol}$ ) added. The reaction was stirred under ambient conditions for a further 6 hours, a further aliquot of DIPEA (100 $\mu 1,0.59 \mathrm{mmol})$ was added and the reaction stored at $3^{\circ} \mathrm{C}$ overnight. Excess activated ester was quenched by addition of $n$-butylamine $(190 \mu$, $1.92 \mathrm{mmol})$ and stirring at room temperature for 1 hour. The reaction mixture was diluted with ethyl acetate (250 $\mathrm{ml})$ and washed successively with aqueous saturated $\mathrm{NaHCO}_{3}(2 \times 150 \mathrm{ml})$ and saturated $\mathrm{NaCl}(2 \times 150 \mathrm{ml})$. The organics were dried over anhydrous sodium sulfate, filtered and solvent removed in vacuo. The resultant red gum was purified by silica gel column chromatography, eluting with a gradient of $0-2 \%(\mathrm{v} / \mathrm{v})$ methanol in DCM containing $1 \%(\mathrm{v} / \mathrm{v})$ triethylamine to give the title compound as an orange solid $(250 \mathrm{mg}, 72 \%)$. ${ }^{1} \mathrm{H}$ NMR $(300$ $\left.\mathrm{MHz}, \mathrm{CDCl}_{3}, 20^{\circ} \mathrm{C}, \mathrm{TMS}\right) \delta=3.40\left(2 \mathrm{H}, \mathrm{m}, \underline{\mathrm{H}} 5\right.$ ', $\left.\underline{\mathrm{H}} 5^{\prime \prime}\right), 3.77\left(6 \mathrm{H}, \mathrm{s}, 2 \mathrm{xOC}^{\mathrm{H}} 3\right), 4.22\left(1 \mathrm{H}, \mathrm{s}, \underline{\mathrm{H}} 4^{\prime}\right), 4.62\left(1 \mathrm{H}, \mathrm{d}^{3} J\right.$ $\left.(\mathrm{H}, \mathrm{H})=4.8 \mathrm{~Hz}, \underline{\mathrm{H}} 2^{\prime}\right), 4.89\left(1 \mathrm{H}, \mathrm{m}, \underline{\mathrm{H}} 3^{\prime}\right), 5.43\left(1 \mathrm{H}, \mathrm{d}^{3} J(\mathrm{H}, \mathrm{H})=8.1 \mathrm{~Hz}, \underline{\mathrm{H}} 5\right), 6.28\left(1 \mathrm{H}, \mathrm{d}^{3} J(\mathrm{H}, \mathrm{H})=8.4 \mathrm{~Hz}\right.$, $\left.\underline{\mathrm{H}}{ }^{\prime}\right), 6.84\left(4 \mathrm{H}, \mathrm{d}^{3} J(\mathrm{H}, \mathrm{H})=9.0 \mathrm{~Hz}, \mathrm{DMTr}-\underline{\mathrm{H}} o-\right.$ to OMe $), 7.20-7.33$ (II7H, m, DMTr- $\left.\underline{\mathrm{H}}, \mathrm{NHC}(\mathrm{O}) \mathrm{AB}\right), 7.40-$ $7.46(6 \mathrm{H}, \mathrm{m}, \mathrm{DMTr}-\underline{\mathrm{H}}, \mathrm{AB}-\underline{\mathrm{H}}), 7.58\left(1 \mathrm{H}, \mathrm{d}^{3} J(\mathrm{H}, \mathrm{H})=7.5 \mathrm{~Hz}, \mathrm{AB}-\underline{\mathrm{H}}\right), 7.67\left(1 \mathrm{H},{ }^{3} J(\mathrm{H}, \underline{\mathrm{H}})=8.1 \mathrm{~Hz}, \underline{\mathrm{H}} 6\right), 7.80-$ $7.94(4 \mathrm{H}, \mathrm{m}, \mathrm{AB}-\underline{\mathrm{H}}), 8.30(1 \mathrm{H}, \mathrm{s}, \mathrm{AB}-\underline{\mathrm{H}}) ;{ }^{13} \mathrm{C} \mathrm{NMR}\left(101 \mathrm{MHz}, \mathrm{CDCl}_{3}, 20^{\circ} \mathrm{C}, \mathrm{TMS}\right) \delta=55.7,58.0,64.1,72.2$, $86.1,86.9,87.6,103.6,113.8 ; 122.3 ; 123.4 ; 127.6 ; 128.5 ; 128.6 ; 129.5 ; 129.8 ; 129.9 ; 130.6 ; 131.8 ; 134.7$; $135.5 ; 135.7 ; 140.5 ; 144.5 ; 151.9 ; 152.8 ; 152.9 ; 159.1 ; 163.5 ; 167.9$; HRMS (ESI) 753.2791 (calc.753.2799 for $\left.\mathrm{MH}^{+}\right)$ 
5'-O-(4,4'-dimethoxytrityl)-2'-(N-(m-phenylazo)benzamido)-2'-deoxyuridine-3'- $O$-(2-cyanoethyl- $N, N$ -

diisopropylamino)-phosphoramidite.

To a stirred solution of $5^{\prime}-O-\left(4,4^{\prime}-\right.$ dimethoxytrityl $)-2^{\prime}-(N-(m-$ phenylazo)benzamido)-2'-deoxyuridine (134 $\mathrm{mg}, 0.18 \mathrm{mmol})$ and DIPEA $(67 \mu \mathrm{l}, 0.73 \mathrm{mmol})$ in anhydrous DCM $(3 \mathrm{ml})$ and THF $(0.5$ $\mathrm{ml})$ at room temperature was added 2-cyanoethyl- $N, N$ diisopropylaminochlorophosphoramidite $(57 \mu 1,0.36 \mathrm{mmol})$ dropwise over $3 \mathrm{~min}$. The reaction was maintained under these conditions for a

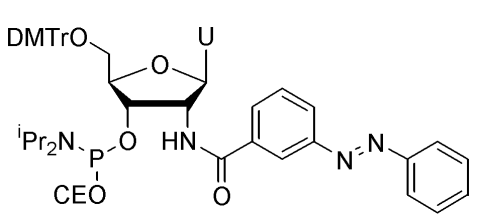
further $30 \mathrm{~min}$ and then quenched following addition of anhydrous methanol (90 $\mu \mathrm{l}, 2.22 \mathrm{mmol})$ and DIPEA (90 $\mu 10.98 \mathrm{mmol})$ and stirring for a further $10 \mathrm{~min}$. The reaction mixture was diluted with acid-free ethyl acetate (50 $\mathrm{ml})$ and washed successively with aqueous saturated $\mathrm{NaHCO}_{3}(2 \times 50 \mathrm{ml})$, saturated $\mathrm{NaCl}(1 \times 50 \mathrm{ml})$. The organics were dried over anhydrous sodium sulfate, filtered and solvent removed in vacuo. The resultant solid was purified by silica gel column chromatography, eluting with $2 \%(\mathrm{v} / \mathrm{v})$ solutions of triethylamine in $3: 1$ DCM:40-60 petroleum ether; pure DCM and 3:1 DCM : ethyl acetate. The title compound was isolated as an orange foam (160 mg, $0.17 \mathrm{mmol}, 93 \%)$. ${ }^{1} \mathrm{H} \mathrm{NMR}\left(300 \mathrm{MHz}, \mathrm{CDCl}_{3}, 20^{\circ} \mathrm{C}\right.$, TMS) $\delta=1.00-1.35$ (II12H, m $2 \mathrm{x}$ $\left.\left(\mathrm{C}_{3}\right)_{2}\right), 2.31$ and $2.44\left(2 \mathrm{H}, 2 \times m, \underline{C}_{2} \mathrm{CN}\right) 3.44\left(2 \mathrm{H}, \mathrm{m}, \underline{\mathrm{H}} 5\right.$ ', $\left.\underline{\mathrm{H}} 5^{\prime \prime}\right) 3.45-3.80\left(4 \mathrm{H}, \mathrm{m},(-\mathrm{C} \underline{\mathrm{H}})_{2} \mathrm{~N}, \mathrm{C}_{2} \mathrm{O}\right), 3.80$ $\left(6 \mathrm{H}, \mathrm{s}, 2 \mathrm{xCH}_{3} \mathrm{O}\right), 4.30$ and $4.45\left(1 \mathrm{H}, 2 \mathrm{xs}, \underline{\mathrm{H}} 4^{\prime}\right), 4.56$ and $4.75\left(1 \mathrm{H}, 2 \mathrm{xdd}, \underline{\mathrm{H}} 2^{\prime}\right), 4.97$ and 5.13 (1H, 2xm, $\left.\underline{\mathrm{H}} 3^{\prime}\right)$, $5.50(1 \mathrm{H}, \psi \mathrm{t}, \underline{\mathrm{H}} 5), 6.26\left(1 \mathrm{H}, \psi \mathrm{t}, \underline{\mathrm{H}} 1^{\prime}\right), 6.87\left(4 \mathrm{H}, 2 \mathrm{xd}^{3} \mathrm{~J}(\mathrm{H}, \mathrm{H})=8.9 \mathrm{~Hz}, \mathrm{DMTr} \underline{\mathrm{H}} o\right.$ - to OMe $), 6.97$ and $7.14(1 \mathrm{H}$,

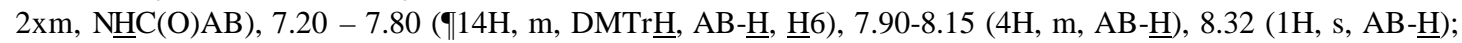
${ }^{31} \mathrm{P} \mathrm{NMR}\left(121 \mathrm{MHz}, \mathrm{CDCl}_{3}, 20^{\circ} \mathrm{C}, 85 \% \mathrm{H}_{3} \mathrm{PO}_{4}\right) \delta=149.4,148.1$

p-Phenylazobenzoic acid- $N$-hydroxysuccinimidyl ester.

To a stirred suspension of $p$-phenylazobenzoic acid $(452 \mathrm{mg}, 2.0 \mathrm{mmol})$ and $\mathrm{N}$-hydroxysuccinimide (255 mg, $2.2 \mathrm{mmol})$ in anhydrous DCM $(10 \mathrm{ml}) \mathrm{N}, \mathrm{N}^{\prime}$ dicyclohexylcarbodiimide (454 mg, $2.2 \mathrm{mmol}$ ) was added at room temperature under argon. After $45 \mathrm{~min}$, the precipitated urea was removed by filtration and the solvent removed in vacuo. The residual orange solid was

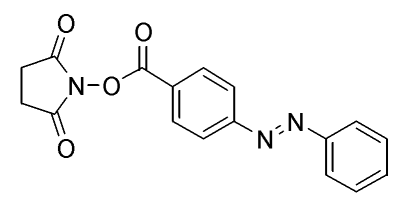
purified by silica gel column chromatography eluting with anhydrous DCM to yield the pure product as a moisture-sensitive orange solid (544 mg, 84\%). ${ }^{1} \mathrm{H}$ NMR $\left(300 \mathrm{MHz}, \mathrm{CDCl}_{3}, 20^{\circ} \mathrm{C}\right.$, TMS $) \delta=2.94(4 \mathrm{H}, \mathrm{s}, 2 \mathrm{x}$ $\left.\mathrm{C}_{2}\right), 7.55(3 \mathrm{H}, \mathrm{m}, \mathrm{Ph} \underline{\mathrm{H}}), 7.97\left(2 \mathrm{H}, \mathrm{m}, \mathrm{Ph} \underline{\mathrm{H}} o\right.$ to $\left.\mathrm{N}_{2}\right), 8.02\left(2 \mathrm{H}, \mathrm{d}^{3} J(\mathrm{H}, \mathrm{H})=8.4 \mathrm{~Hz} \operatorname{Ar} \underline{\mathrm{H}} o\right.$ to $\left.\mathrm{C}(\mathrm{O})\right), 8.30,(2 \mathrm{H}, \mathrm{d}$ ${ }^{3} J(\mathrm{H}, \mathrm{H})=8.4 \mathrm{~Hz}, \operatorname{Ar} \underline{\mathrm{H}} o$ to $\left.\mathrm{N}_{2}\right),{ }^{13} \mathrm{C} \mathrm{NMR}\left(101 \mathrm{Mz}, \mathrm{CDCl}_{3}, 20^{\circ} \mathrm{C}, \mathrm{TMS}\right) \delta=25.7,123.0,123.3,126.5,129.2$, $131.7,132.1,152.4,156.1,161.4,169.2 ; \mathrm{CI}+\mathrm{MS} \mathrm{m} / \mathrm{z} 324$ (MH+)

5'-O-(4,4'-dimethoxytrityl)-2'-( $N$-( $p$-phenylazo)benzamido)-2'-deoxyuridine.

To a stirred solution of $p$-phenylazobenzoic acid- $N$ - DMTrO hydroxysuccinimidyl ester $(260 \mathrm{mg}, 0.80 \mathrm{mmol})$ in anhydrous DMF (5 $\mathrm{ml})$ at room temperature was added $n$-butylamine $(10 \mu 1,0.10 \mathrm{mmol})$ and the reaction mixture maintained under these conditions for a further $10 \mathrm{~min}$. The reaction mixture was transferred under argon to a<smiles>CCC(O)C(O)C(Cl)NC(=O)c1ccc(N=Nc2ccccc2)cc1</smiles>
flask containing 5'-O-(4,4'-dimethoxytrityl)-2'-amino-2'-deoxyuridine $(250 \mathrm{mg}, 0.46 \mathrm{mmol})$ and $\mathrm{N}, \mathrm{N}$ diisopropylethylamine (DIPEA: $80 \mu 1,0.47 \mathrm{mmol}$ ) added. The reaction was stirred under ambient conditions for a further 6 hours, a further aliquot of DIPEA $(100 \mu \mathrm{l}, 0.59 \mathrm{mmol})$ was added and the reaction stored at $3^{\circ} \mathrm{C}$ overnight. Excess activated ester was quenched by addition of $n$-butylamine $(190 \mu 1,1.92 \mathrm{mmol})$ and stirring at room temperature for 1 hour. The reaction mixture was diluted with ethyl acetate $(250 \mathrm{ml})$ and washed successively with aqueous saturated $\mathrm{NaHCO}_{3}(2 \times 150 \mathrm{ml})$ and saturated $\mathrm{NaCl}(2 \times 150 \mathrm{ml})$. The organics were dried over anhydrous sodium sulfate, filtered and solvent removed in vacuo. The resultant red gum was purified by silica gel column chromatography, eluting with a gradient of $0-2 \%(\mathrm{v} / \mathrm{v})$ methanol in DCM containing $1 \%$ (v/v) triethylamine to give the title compound as an orange solid $(250 \mathrm{mg}, 72 \%) .{ }^{1} \mathrm{H} \mathrm{NMR} \mathrm{(300} \mathrm{MHz}, \mathrm{CDCl}_{3}$, $\left.20^{\circ} \mathrm{C}, \mathrm{TMS}\right) \delta=3.42\left(2 \mathrm{H}, \mathrm{m}, \underline{\mathrm{H}} 5^{\prime}, \underline{\mathrm{H}} 5^{\prime \prime}\right), 3.75\left(6 \mathrm{H}, \mathrm{s}, 2 \mathrm{xOCH}_{3}\right), 4.23\left(1 \mathrm{H}, \mathrm{s}, \underline{\mathrm{H}} 4^{\prime}\right), 4.65\left(1 \mathrm{H}, \mathrm{m}, \underline{\mathrm{H}} 2^{\prime}\right), 4.77(1 \mathrm{H}$, $\left.\mathrm{m}, \underline{\mathrm{H}} 3^{\prime}\right), 5.47\left(1 \mathrm{H}, \mathrm{d}^{3} J(\mathrm{H}, \mathrm{H})=8.2 \mathrm{~Hz}, \underline{\mathrm{H}} 5\right), 6.20\left(1 \mathrm{H}, \mathrm{d}^{3} J(\mathrm{H}, \mathrm{H})=8.1 \mathrm{~Hz}, \underline{\mathrm{H}} 1^{\prime}\right), 6.89\left(4 \mathrm{H}, \mathrm{d}^{3} J(\mathrm{H}, \mathrm{H})=9.0 \mathrm{~Hz}\right.$, DMTr- $\underline{\mathrm{H}} o$ - to OMe), 7.20 - $7.43(\mathrm{I} 10 \mathrm{H}, \mathrm{m}, \mathrm{DMTr}-\underline{\mathrm{H}}, \mathrm{NHC}(\mathrm{O}) \mathrm{AB}) .7 .48(3 \mathrm{H}, \mathrm{m}, \mathrm{AB}-\underline{\mathrm{H}}) 7.67\left(1 \mathrm{H}, \mathrm{d},{ }^{3} J(\mathrm{H}, \mathrm{H})\right.$ $=8.2 \mathrm{~Hz}, \underline{\mathrm{H}} 6), 7.85-7.95(6 \mathrm{H}, \mathrm{m}, \mathrm{AB}-\underline{\mathrm{H}}) ;{ }^{13} \mathrm{C} \mathrm{NMR}\left(63 \mathrm{MHz}, \mathrm{CDCl}_{3}, 20^{\circ} \mathrm{C}, \mathrm{TMS}\right) \delta=55.7,57.9,64.2,72.1$, 86.3, 87.1, 87.6, 103.6, 113.8, 123.3, 123.6, 127.6, 128.5, 128.6, 128.7, 129.5, 130.6, 132.0, 135.4, 135.6, $135.7,140.5,144.5,152.0,152.9,154.8,159.1,163.7,167.8 ; \mathrm{FAB}+\mathrm{MS}$ m/z 754 (MH+) 
5'-O-(4,4'-dimethoxytrityl)-2'-( $N$-(p-phenylazo)benzamido)-2'-deoxyuridine-3'- $O$-(2-cyanoethyl- $N, N$ diisopropylamino)-phosphoramidite. To a stirred solution of $5^{\prime}-O-\left(4,4^{\prime}-\right.$ dimethoxytrityl)-2'-( $N-(p-$ phenylazo)benzamido)-2'-deoxyuridine (250 $\mathrm{mg}, 0.33 \mathrm{mmol})$ and DIPEA $(125 \mu \mathrm{l}, 1.36 \mathrm{mmol})$ in anhydrous DCM $(5 \mathrm{ml})$ and THF $(2$ $\mathrm{ml})$ at room temperature was added 2-cyanoethyl- $N, N$ diisopropylaminochlorophosphoramidite $(106 \mu 1, \quad 0.66 \quad \mathrm{mmol})$<smiles>C=[PH](NC(C)C)OC1C(COC)OC(Cl)C1NC(=O)c1ccc(N=Nc2ccccc2)cc1</smiles>
dropwise over $3 \mathrm{~min}$. The reaction was maintained under these conditions for a further $30 \mathrm{~min}$ and then quenched following addition of anhydrous methanol $(60 \mu \mathrm{l}, 1.48 \mathrm{mmol})$ and DIPEA $(60 \mu \mathrm{l} 0.65 \mathrm{mmol})$ and stirring for a further $10 \mathrm{~min}$. The reaction mixture was diluted with acid-free ethyl acetate $(250 \mathrm{ml})$ and washed successively with aqueous saturated $\mathrm{NaHCO}_{3}(2 \times 250 \mathrm{ml})$, saturated $\mathrm{NaCl}(1$ $\mathrm{x} 150 \mathrm{ml}$ ). The organics were dried over anhydrous sodium sulfate, filtered and solvent removed in vacuo. The resultant solid was purified by silica gel column chromatography, eluting with $2 \%(\mathrm{v} / \mathrm{v})$ solutions of triethylamine in 3:1 DCM: 40-60 petroleum ether; pure DCM and 3:1 DCM : ethyl acetate. The title compound was isolated as an orange foam (308 mg, $0.32 \mathrm{mmol}, 97 \%)$. ${ }^{1} \mathrm{H} \mathrm{NMR}\left(300 \mathrm{MHz}, \mathrm{CDCl}_{3}, 20^{\circ} \mathrm{C}, \mathrm{TMS}\right) \delta=0.95-$ $1.25\left(\mathrm{I}\left[12 \mathrm{H}, \mathrm{m} 2 \times\left(\mathrm{CH}_{3}\right)_{2}\right), 2.33\right.$ and $2.48\left(2 \mathrm{H}, 2 \times \mathrm{m}, \mathrm{CH}_{2} \mathrm{CN}\right) 3.36\left(2 \mathrm{H}, \mathrm{m}, \underline{\mathrm{H}} 5\right.$ ', $\left.\underline{\mathrm{H}} 5^{\prime \prime}\right) 3.40-3.80$ (4H, m, ($\left.\mathrm{CH})_{2} \mathrm{~N}, \mathrm{CH}_{2} \mathrm{O}\right), 3.80\left(6 \mathrm{H}, \mathrm{s}, 2 \mathrm{xCH}_{3} \mathrm{O}\right), 4.30$ and $4.46\left(1 \mathrm{H}, 2 \mathrm{xs}, \underline{\mathrm{H}} 4^{\prime}\right), 4.53$ and $4.69\left(1 \mathrm{H}, 2 \mathrm{xdd}^{3} J(\mathrm{H}, \mathrm{H})=6.8 \mathrm{~Hz}\right.$, $\left.\underline{\mathrm{H}} 2^{\prime}\right), 4.97$ and $5.09\left(1 \mathrm{H}, 2 \mathrm{xm}, \underline{\mathrm{H}} 3\right.$ '), $5.49(1 \mathrm{H}, \psi \mathrm{t}, \underline{\mathrm{H}} 5), 6.22$ and $6.29\left(1 \mathrm{H}, 2 \mathrm{xd}^{3} J(\mathrm{H}, \mathrm{H})=6.8 \mathrm{~Hz}, \mathrm{H} 1^{\prime}\right), 6.82$ and $6.83\left(4 \mathrm{H}, 2 \mathrm{xd}^{3} J(\mathrm{H}, \mathrm{H})=6.8 \mathrm{~Hz}, \mathrm{DMTr} \underline{\mathrm{H}} o\right.$ - to $\left.\mathrm{OMe}\right), 6.97$ and $7.14(1 \mathrm{H}, 2 \mathrm{xm}, \mathrm{N} \underline{\mathrm{HC}}(\mathrm{O}) \mathrm{AB}), 7.30-7.55$ (II $12 \mathrm{H}, \mathrm{m}, \mathrm{DMTr} \underline{\mathrm{H}}, \mathrm{AB}-\underline{\mathrm{H}}), 7.72$ and $7.81\left(1 \mathrm{H}, 2 \mathrm{xd}{ }^{3} \mathrm{~J}(\mathrm{H}, \mathrm{H})=8.2 \mathrm{~Hz}, \underline{\mathrm{H}} 6\right), 7.85-7.90(6 \mathrm{H}, \mathrm{m}, \mathrm{AB}-\underline{\mathrm{H}}) ;{ }^{31} \mathrm{P}$ NMR $\left(101 \mathrm{MHz}, \mathrm{CDCl}_{3}, 85 \% \mathrm{H}_{3} \mathrm{PO}_{4}\right) \delta=152.1,149.8 ; \mathrm{FAB}+\mathrm{MS} \mathrm{m} / \mathrm{z} 853\left(\mathrm{M}^{\mathrm{i}} \mathrm{Pr}_{2} \mathrm{~N}\right), 883$ (M-OCE), 954 $(\mathrm{M}+\mathrm{H})$

\section{Synthesis of oligonucleotides}

Oligosynthesis was carried out on Beckman Oligo 1000M and Expedite 8909 DNA/RNA synthesizers on a 1 Amol scale using standard DNA and RNA phosphoramidites from Link Technologies. Standard nucleobase protecting groups and 2'- $O$-TBDMS-protection were used. Benzylthiotetrazole $(0.2 \mathrm{M})$ was used as activating agent. Oxidation was performed using 8:1:1 THF:pyridine: $\mathrm{H}_{2} \mathrm{O}$ containing either $50 \mathrm{mM}$ or $20 \mathrm{mM} \mathrm{I}_{2}$ for, respectively, Beckman- or Expedite-run syntheses. Unmodified DNA and RNA were synthesized DMT-on and deprotected and purified according to standard procedures. The coupling time was extended (5-6 min) for 2'azobenzene-modified amidites. Deprotection of azobenzene-modified oligonucleotides was performed with 1.5 $\mathrm{ml}$ of an anhydrous mixture of $\mathrm{NH}_{3} / \mathrm{MeOH}$ (dry $\mathrm{MeOH}$ saturated with gaseous ammonia at $-10^{\circ} \mathrm{C}$ ) overnight at room temperature. The decanted supernatant was evaporated and the crude oligonucleotides purified by RPHPLC (column: Hichrom KR100-5C18; buffer A: 0.1 M TEAAc, pH 6.5, 5\% (v/v) MeCN; buffer B: $0.1 \mathrm{M}$ TEAAc, pH 6.5, 65\% (v/v) MeCN) monitoring absorbance in the range $200-400$ (or 600) $\mathrm{nm}$ with a diode array detector. After evaporation of the buffer the oligonucleotides were desalted using standard SepPak protocols (Waters) and concentrations determined using UV absorbance at $260 \mathrm{~nm}$.

\section{MALDI-TOF analysis of DNA-azobenzene conjugates}

\begin{tabular}{|l|l|}
\hline DNA oligomer & Mass (calc.) \\
\hline DRo & $8438.79(8440.46)$ \\
\hline DRm & $8434.24(8440.46)$ \\
\hline DRp & $8434.17(8440.46)$ \\
\hline ACC1oGGTA & $2620.29(2617.51)$ \\
\hline ACC1mGGTA & $2619.58(2617.51)$ \\
\hline ACC1pGGTA & $2626.46(2617.51)$ \\
\hline
\end{tabular}

\section{Photoswitching of DNA-azobenzene conjugates}

For the irradiation a Medium Pressure Hg Arc-lamp (100 W, Engelhard Hanovia of Canada Ltd.) was utilized. A band-pass filter was used for irradiation at $366 \mathrm{~nm}(4.13 \mathrm{~W})$ and a cutoff filter was used for irradiation $>400 \mathrm{~nm}$ $(435 \mathrm{~nm}, 3.36 \mathrm{~W})$ in combination with a water filter $(1 \mathrm{~cm})$ to prevent warming of the samples during prolonged irradiation.

As the high concentrations of deoxyribozyme and labeled substrate used prevented full kinetic analysis, all constructs were assayed at a fixed set of standard conditions ( $20 \mu \mathrm{M}$ substrate, $2 \mu \mathrm{M}$ deoxyribozyme), and from the measured initial rates, apparent first order rate constants of the $Z$ - and $E$-isomers were determined. 


\section{ACC1oGGTA}

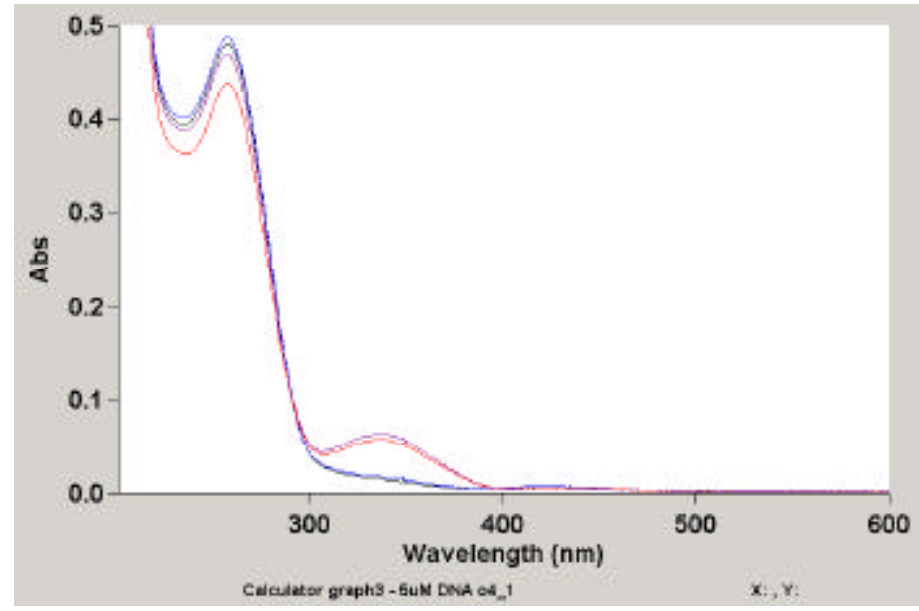

\section{$\underline{\text { KEY }}$}

- UV spectrum of 8-mer following HPLC purification

- UV spectrum of 8-mer following irradiation at $366 \mathrm{~nm}$ for $8 \mathrm{~min}$

- UV spectrum of 8-mer following irradiation at $366 \mathrm{~nm}$ for $8 \mathrm{~min}$ and incubation at $26^{\circ} \mathrm{C}$ for $6 \mathrm{~h}$

- UV spectrum of 8-mer following irradiation at $435 \mathrm{~nm}$ for $2 \mathrm{~min}$

\section{ACC1mGGTA}

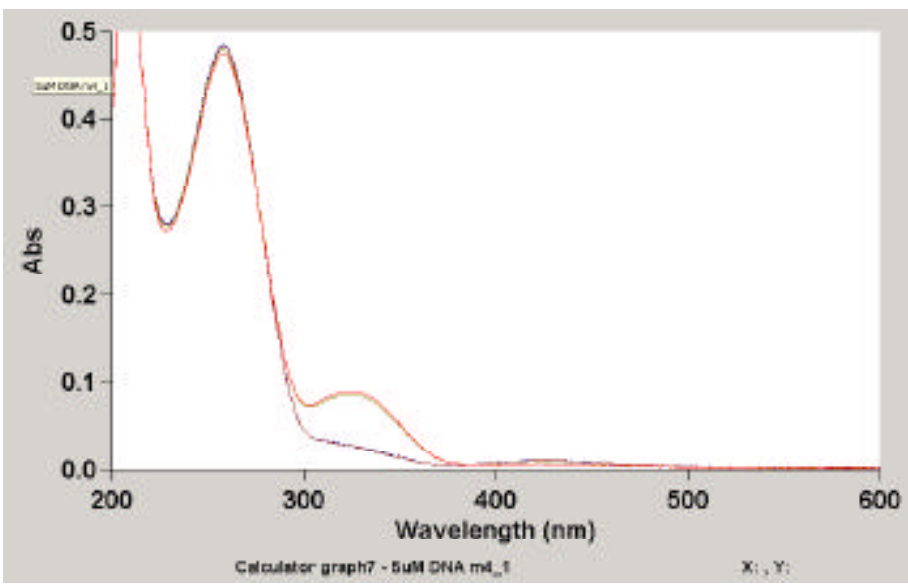

$\underline{\text { KEY }}$

- UV spectrum of 8-mer following HPLC purification

- UV spectrum of 8-mer following irradiation at $366 \mathrm{~nm}$ for $8 \mathrm{~min}$

- UV spectrum of 8-mer following irradiation at $366 \mathrm{~nm}$ for $8 \mathrm{~min}$ and incubation at $26^{\circ} \mathrm{C}$ for $6 \mathrm{~h}$

- UV spectrum of 8-mer following irradiation at $435 \mathrm{~nm}$ for $2 \mathrm{~min}$

\section{KEY}

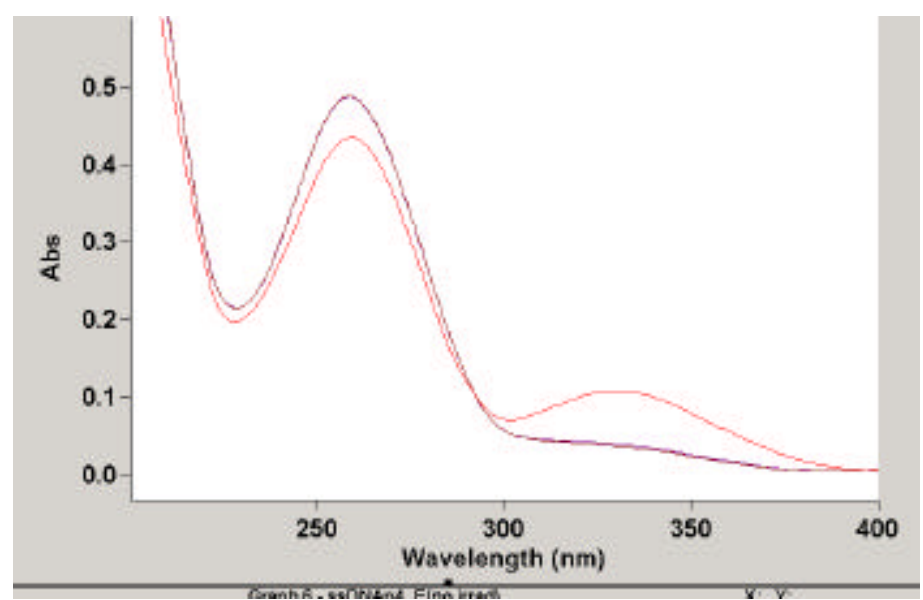

- UV spectrum of 8-mer following HPLC purification

- UV spectrum of 8-mer following irradiation at $366 \mathrm{~nm}$ for $5 \mathrm{~min}$

- UV spectrum of 8-mer following irradiation at $366 \mathrm{~nm}$ for a further $3 \mathrm{~min}$ 
Conditions for DRm and DRp: Hichrom KR100-5C18 with UV detection eluting with buffer A: 0.1 M TEAAc, pH 6.5, solvent C: MeCN, monitored at $260 \mathrm{~nm}$; gradient indicated by purple line

Conditions for ACC1oGGTA: Hichrom KR100-5C18 eluting with buffer A: 0.1 M TEAAc, pH 6.5, buffer B: 0.1 M TEAAc, $\mathrm{pH} 6.5,65 \%(\mathrm{v} / \mathrm{v}) \mathrm{MeCN}$, monitored at $260 \mathrm{~nm}$; gradient indicated by turquoise line.

\section{DRm}

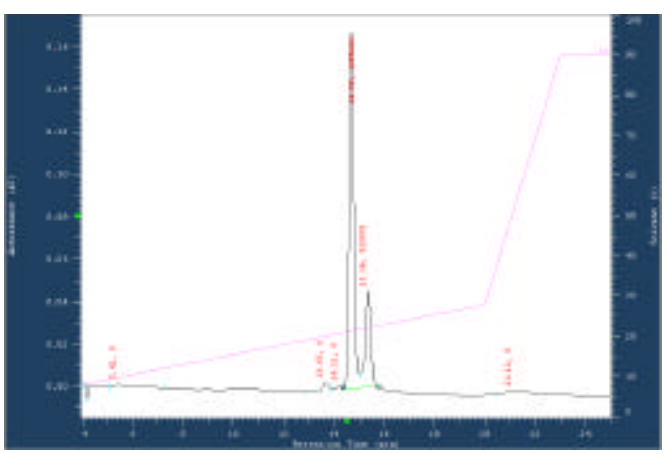

DRp

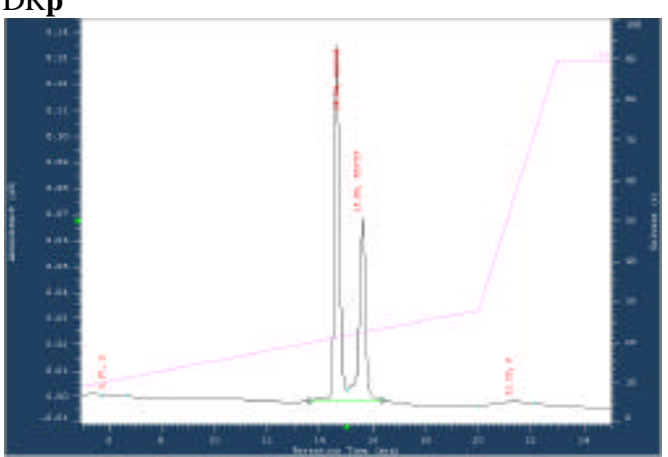

ACC1oGGTA

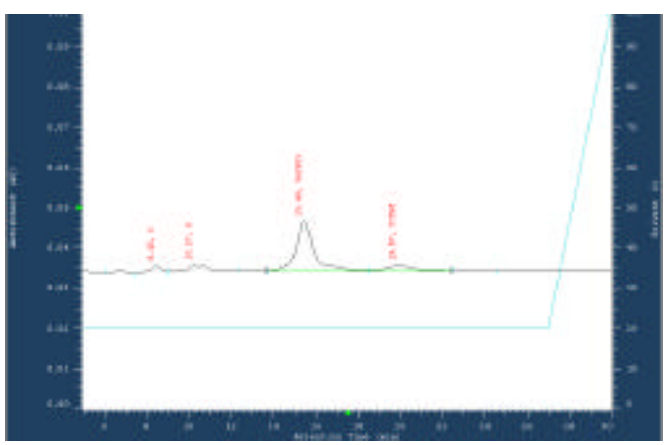

$\underline{\mathrm{KEY}}$

$14.69 \min Z$-DRm $(75 \%)$

$15.36 \min E$-DRm $(25 \%)$

\section{$\underline{\mathrm{KEY}}$}

$14.64 \min Z$-DRp $(61 \%)$

$15.60 \min E$-DRp (39\%)

\section{$\underline{\mathrm{KEY}}$}

15.44 min Z-ACC1oGGTA (86\%)

19.87 min $E$-ACC1oGGTA (14\%) 


\section{Reversible photocontrol of deoxyribozyme-catalyzed RNA cleavage under multiple turnover conditions}

\section{Activity assays for 10-23 deoxyribozymes in the PAGE format}

For the measurements of RNA-cleavage activities under multiple turnover conditions the deoxyribozyme construct (final concentration $2 \mu \mathrm{M}$ or $1 \mu \mathrm{M}$ ) was added to a buffered solution containing $50 \mathrm{mM}$ Tris, $\mathrm{pH} 7.5,40$ $\mathrm{mM} \mathrm{NaCl}$ and $25 \mathrm{mM} \mathrm{MgCl} 2$ (all final concentrations). Preirradiation of these mixtures was performed at 366 $\mathrm{nm}$ for $10 \mathrm{~min}$ and at $435 \mathrm{~nm}$ for $2 \mathrm{~min}$ in clear eppendorf tubes. The reaction was initiated by addition of the deoxyribozyme solutions to the substrate RNA $(20 \mu \mathrm{M})$, yielding a total volume of 50 or $100 \mu \mathrm{l}$. Incubation was performed in the absence of light at $26^{\circ} \mathrm{C}$, except for irrDRp, which was incubated under continuous irradiation at $366 \mathrm{~nm}$ (temperature under these conditions was determined to be $26^{\circ} \mathrm{C}$ ). After appropriate time intervals aliquots $(10 \mu \mathrm{l})$ were withdrawn, mixed with a stop solution $\left(6 \mathrm{M}\right.$ urea, $50 \mathrm{mM}$ EDTA) and frozen at $-20^{\circ} \mathrm{C}$. The samples were loaded on a $15 \%(\mathrm{w} / \mathrm{v})(3 \%$ cross-linked) denaturing polyacrylamide sequencing gel and run at 900 $\mathrm{V}$ for $2 \mathrm{hr}$. The gel was then removed from the plates and transferred onto a gel documentation system (Versadoc, Biorad) and analyzed. Quantitation of the bands was performed with the accompanying software (QuantityOne) such that the intensity of the product bands was set in relation to the sum of the intensities of the product and substrate bands which resulted in relative cleavage values.

\section{$\underline{\text { Activity assays for 10-23 deoxyribozymes using RP-HPLC analysis }}$}

For the measurements of RNA-cleavage activities under multiple turnover conditions the deoxyribozyme

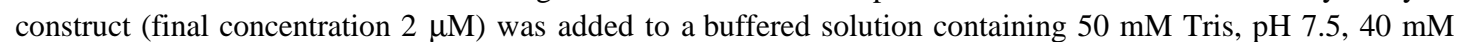
$\mathrm{NaCl}$ and $25 \mathrm{mM} \mathrm{MgCl}_{2}$ (all final concentrations). Preirradiation of these mixtures was performed at $366 \mathrm{~nm}$ for $10 \mathrm{~min}$ and at $435 \mathrm{~nm}$ for $2 \mathrm{~min}$ in clear eppendorf tubes. The reaction was initiated by addition of the deoxyribozyme solutions to the substrate RNA $(20 \mu \mathrm{M})$, yielding a total volume of $50 \mu \mathrm{l}$. Incubation was performed in the absence of light at $26^{\circ} \mathrm{C}$, except for $\operatorname{irrDRp}$, which was incubated under continuous irradiation at $366 \mathrm{~nm}$ (temperature under these conditions was determined to be $26^{\circ} \mathrm{C}$ ). After $1.5 \mathrm{hr}$ an aliquot $(45 \mu \mathrm{l})$ was removed and quenched with $10 \mu \mathrm{l} 250 \mathrm{mM}$ EDTA. For the injection $40 \mu \mathrm{l} 0.1 \mathrm{M}$ TEAAc were added and the samples analyzed on RP-HPLC (column: Hichrom KR100-5C18, buffer A: 0.1 M TEAAc, pH 6.5; buffer B: 0.1 M TEAAc, $\mathrm{pH} 6.5,65 \%(\mathrm{v} / \mathrm{v}) \mathrm{MeCN}$; UV detection at $260 \mathrm{~nm}$; gradient indicated by turquoise line). Quantitation was performed by determining peak areas for the two product peaks and set in relation to the sum of the peak areas of the product and substrate peaks which resulted in relative cleavage values. 
Reversible photocontrol of deoxyribozyme-catalyzed RNA cleavage under multiple turnover conditions

\section{$\underline{\text { HPLC activity assays }}$}

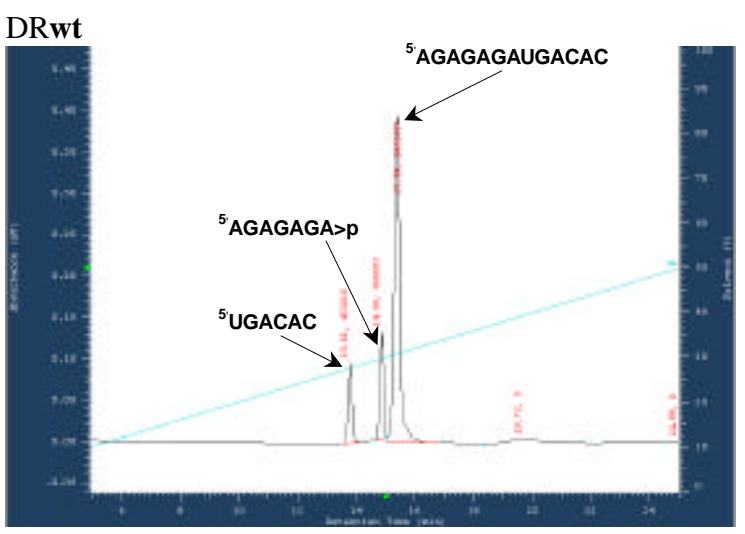

Cleavage of unlabeled RNA substrate after $90 \mathrm{~min}$ by $d-a$ DRs and $i r r$ RRs monitored by HPLC.
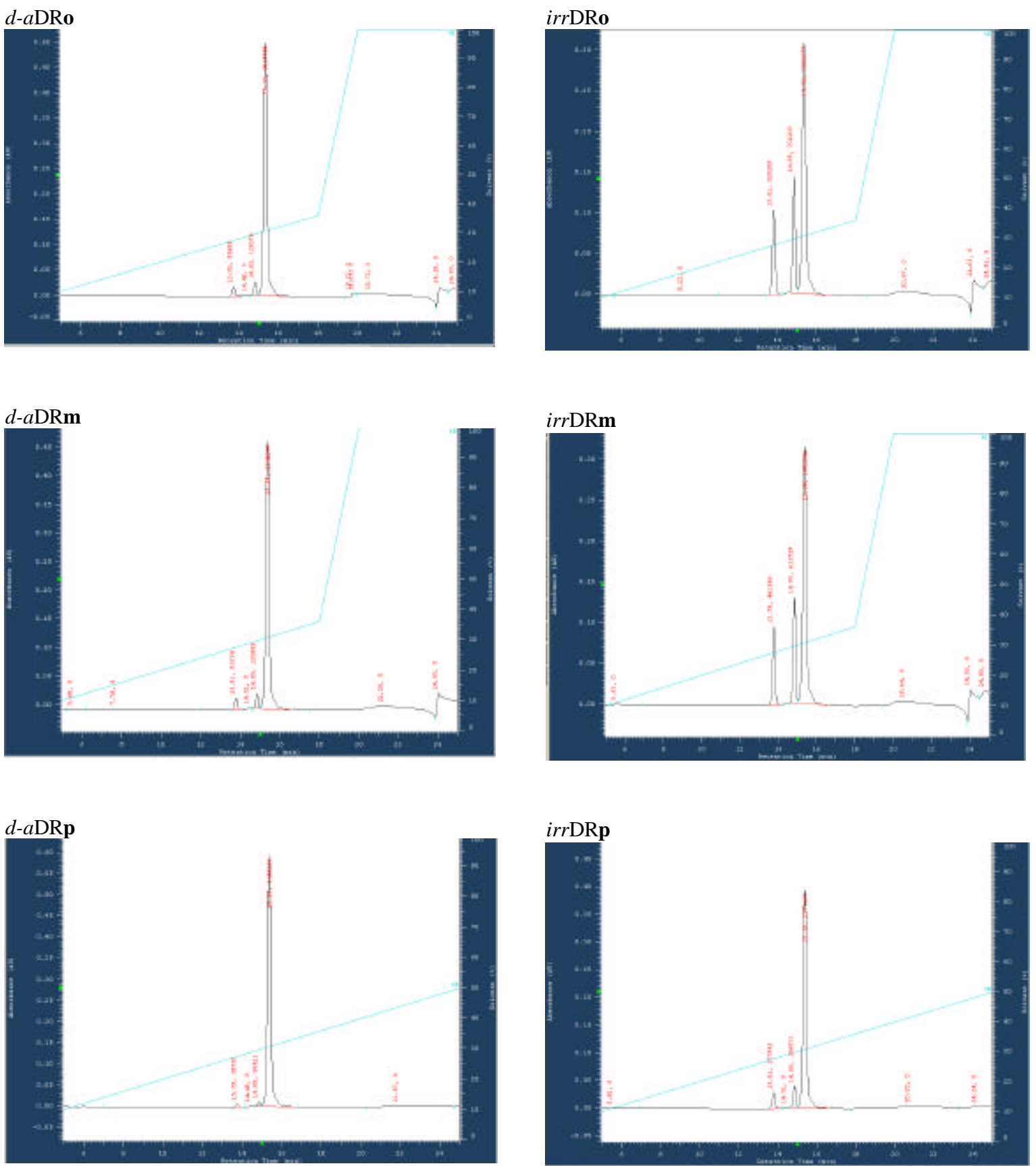